\title{
Cytomegalovirus-associated superior mesenteric vein thrombosis treated with systemic and in-situ thrombolysis Wouter Van Moerkercke ${ }^{\mathrm{a}}$, Karen Pauwelyn ${ }^{\mathrm{a}}$, Eddy Brugman ${ }^{\mathrm{b}}$ and Marc Verhamme ${ }^{\mathrm{C}}$
}

\begin{abstract}
A 56-year-old patient, first diagnosed with an acute cytomegalovirus infection, presented with progressive abdominal pain because of a superior mesenteric vein thrombosis for which he was treated with systemic thrombolysis and heparin in continuous infusion. As this therapy did not have the intended success after 5 days, an interventional radiological procedure was performed with local thrombolysis in the superior mesenteric artery resulting in recanalisation of the vein. Oral anticoagulation was initiated and continued for a period of 6 months.

Mesenteric venous thrombosis is a relatively uncommon cause of mesenteric ischemia that can be associated with severe morbidity and significant mortality. With noninvasive techniques, it is possible to establish a diagnosis in the majority of the cases. The importance of an early diagnosis and therapy - not only with anticoagulation, but also thrombolysis in selected cases - is shown with this case
\end{abstract}

\section{Introduction}

Thrombosis of the (superior) mesenteric vein is a serious event and had frequently been diagnosed in a chronic and mostly irreversible state. Indeed, until recently, portal vein $(\mathrm{PV})$ thrombosis was generally recognized at a stage of cavernous transformation complicated with portal hypertension [1]. These days, a threshold to perform a computed tomographic (CT) scan of the abdomen has greatly diminished when a clinician is confronted with a patient with abdominal pain without obvious etiology. As a consequence, acute portomesenteric vein thrombosis (PMVT) has been diagnosed more frequently, allowing more efficient therapy such as thrombolysis.

A hypercoagulable state can be the result of a desequilibrium between coagulation and fibrinolysis [2]. Both congenital and acquired conditions, predisposing to thrombosis, will be discussed. The concept of thrombosis as a multifactorial process is highlighted. With this case report, we first stress the importance of the role of an acute cytomegalovirus (CMV) infection in the pathogenesis. More cases have been reported in the literature worldwide [3-13]. On account of the rarity of reports of CMV associated with venous thromboembolism in general, it is assumed that CMV infection is a trigger to thrombosis in the context of a predisposition to thromboembolic disease [5]. Second, we discuss the diagnostic and (more aggressive) therapeutic approach of PMVT in general. and review of the literature. Eur J Gastroenterol Hepatol 21:587-592 (c) 2009 Wolters Kluwer Health | Lippincott Williams \& Wilkins.

European Journal of Gastroenterology \& Hepatology 2009, 21:587-592

Keywords: cytomegalovirus infection, hypercoagulable state, mesenteric vein thrombosis, portal vein thrombosis, thrombolysis

${ }^{a}$ Department of Gastroenterology and Hepatology, University Hospitals Leuven, Leuven, Departments of ${ }^{\mathrm{b}}$ Radiology and ${ }^{\mathrm{C}}$ Gastroenterology and Hepatology, AZ Groeninge, Kortrijk, Belgium

Correspondence to Dr Wouter Van Moerkercke, MD, Department of Gastroenterology and Hepatology, University Hospitals Leuven, Herestraat 49, Leuven 3000 , Belgium

Tel: + 321634 4225; fax: + 3216344419 ;

e-mail: wouter.vanmoerkercke@scarlet.be

Received 24 June 2008 Accepted 15 September 2008

\section{Case report}

A 56-year-old immunocompetent male patient presented with symtoms of fever up to $38^{\circ} \mathrm{C}$ and persisting cough since several days. Except for known hypertension and hypercholesterolemia, he had a blank medical history. Liver function tests were moderately disturbed, and CMV IgM antibodies were detected. Screening for hepatitis and HIV was negative. He was diagnosed as having an acute CMV infection. Initial treatment was symptomatic and consisted of adequate analgesia and antipyretics.

Ten days after discharge he was readmitted because of anorexia and progressive abdominal pain with diffuse tenderness at clinical examination. A contrast-enhanced CT scan of the abdomen showed a thrombosis of the superior mesenteric vein (SMV) (Fig. 1). Upon upper endoscopic evaluation, no esophageal varices were observed. A screening for thrombophilia (Table 1) revealed the presence of anticardiolipin antibodies and lupus anticoagulans. Janus kinase 2 mutation was negative. In addition, a seroconversion with the appearance of CMV IgG antibodies was confirmed 16 days after the first admission with acute CMV infection.

Systemic thrombolysis with recombinant tissue plasminogen activator (rt-PA; a bolus of $10 \mathrm{mg}$ rt-PA with a continuous infusion of $90 \mathrm{mg}$ in $2 \mathrm{~h}$ ) did not have the intended result. After this procedure, the abdominal pain with tenderness mainly localized in the region of the 
Fig. 1
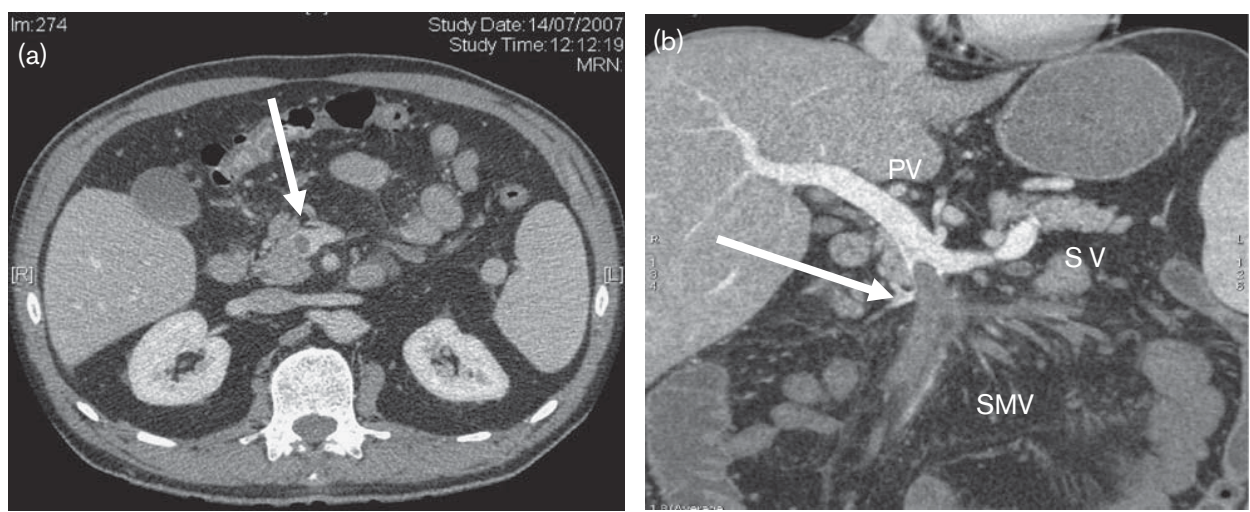

Computer tomography of the abdomen upon admission of the patient, 16 days after the initial diagnosis of an acute cytomegalovirus infection, showing thrombotic material in the superior mesenteric vein [arrow, (a)]. Reconstruction, showing the extent of the thrombus [arrow, (b)] to the confluens of the splenic vein (SV) and superior mesenteric vein (SMV) with protrusion to the portal vein (PV).

Table 1 Results of screening for thrombophilia upon diagnosis of the SMVT in the patient, as described in the case report

\begin{tabular}{lccc}
\hline & Result & Reference & Unit \\
\hline CMV IgG $^{\mathrm{a}}$ & $<4$ & $0-6$ & $\mathrm{IU} / \mathrm{ml}$ \\
$\mathrm{CMV} \mathrm{IgM}^{\mathrm{a}}$ & Positive & Negative & \\
Factor VIII & 183 & $70-120$ & $\%$ \\
vWF & 73 & & $\%$ \\
D-dimers & 5110 & $0-500$ & $\mu \mathrm{g} / \mathrm{l}$ \\
Antithrombin III & 102 & $80-120$ & $\%$ \\
APC-resistance & 121 & $>120$ & $\mathrm{~S}$ \\
Protein C (Act) & 108 & $70-140$ & $\%$ \\
Protein S (Act) & 146 & $70-140$ & $\%$ \\
Anticardiolipin IgG & 29.6 & $<10$ & $\mathrm{GPL} / \mathrm{ml}$ \\
Anticardiolipin IgM & 17.6 & $<10$ & $\mathrm{MPL} / \mathrm{ml}$ \\
Lupus anticoagulans & Positive & & \\
Homocystein & 6.68 & $5-15$ & $\mu \mathrm{mol} / \mathrm{l}$ \\
CMV IgG & 23 & $0-6$ & $\mathrm{IU} / \mathrm{ml}$ \\
CMV IgM & Positive & Negative & \\
Factor V Leiden & Wild type (no mutation) & & \\
Factor II G/A 20210 & Wild type (no mutation) & & \\
Janus kinase 2 mutation & Not present & & \\
\hline
\end{tabular}

Act, activated; APC, activated protein C; CMV, cytomegalovirus; SMVT, superior mesenteric vein thrombosis; vWF, von Willebrand factor.

${ }^{a}$ On day 1 (admission at the Service of Pneumology).

${ }^{b}$ On day 16 (admission at the Service of Gastroenterology and Hepatology).

mesogastrium, persisted with the same intensity. A magnetic resonance angiography, performed 4 days after systemic thrombolysis, showed no thrombotic resolution. Subsequently, it was decided to inject a local thrombolyticum in the superior mesenteric artery (SMA) (Fig. 2a and b). A combination therapy consisting of rt-PA $(0.3 \mathrm{mg} / \mathrm{ml})$, with a total dose of $55 \mathrm{mg}$, and heparin $(133 \mathrm{IU} / \mathrm{ml})$ was initiated at an initial dose of $6 \mathrm{ml} / \mathrm{h}(1.8 \mathrm{mg} \mathrm{rt}-\mathrm{PA} / \mathrm{h})$ and increased to $10 \mathrm{ml} / \mathrm{h}(3 \mathrm{mg}$ $\mathrm{rt}-\mathrm{PA} / \mathrm{h})$. After starting a heparin infusion at $1000 \mathrm{IU} / \mathrm{h}$ oral anticoagulation was associated. A CT relook showed a patent SMV with complete resolution of the thrombus (Fig. 3).

\section{Discussion}

Mesenteric vein thrombosis (MVT) was described as a cause of intestinal ischemia in 1895 by Elliot [14]. Warren and Eberhard differentiated venous thrombosis from arterial thrombosis as a cause of intestinal infarction [14-16]. In up to $95 \%$ of the cases, the SMV is affected.

Table 2 shows a summary of possible etiologies for MVT [2,15,17-22]. Currently, it is possible to identify an etiological factor in three-quarters of the patients [15]. When a clinician is confronted with a new diagnosis of PMVT, one should exclude local factors such as inflammatory processes (pancreatitis,... ), intra-abdominal surgery, intra-abdominal neoplasm, ... that can trigger a PMVT (Table 2). In the section of systemic factors, we use the concept of thrombophilia that can be either congenital or acquired (for the latter, myeloproliferative disorders, such as polycythemia vera and essential thrombocythemia, are known risk factors). With the evolution of better medical care, diagnostic imaging studies, antibiotic treatment, and the better understanding of the coagulation system (in which the importance of congenital disorders is obvious), there is a shift in the profile of possible etiologies for PMVT [20,22]. As mentioned before, thrombophilia can be caused by congenital and acquired factors. In the discussion of inherited thrombophilia (mutations of factor $\mathrm{V}$ Leiden, protrombin, deficiencies of protein C, S, and antitrombin III), it is important to know that both a decreased hepatic synthesis of coagulation factors and activation of coagulation (which is apparent when a PMV'T occurs) lead to a nonspecific decrease of protein $\mathrm{C}, \mathrm{S}$, and antithrombin $[2,15,19]$. This makes it more difficult to know if the deficiency is primary or not. 
Fig. 2
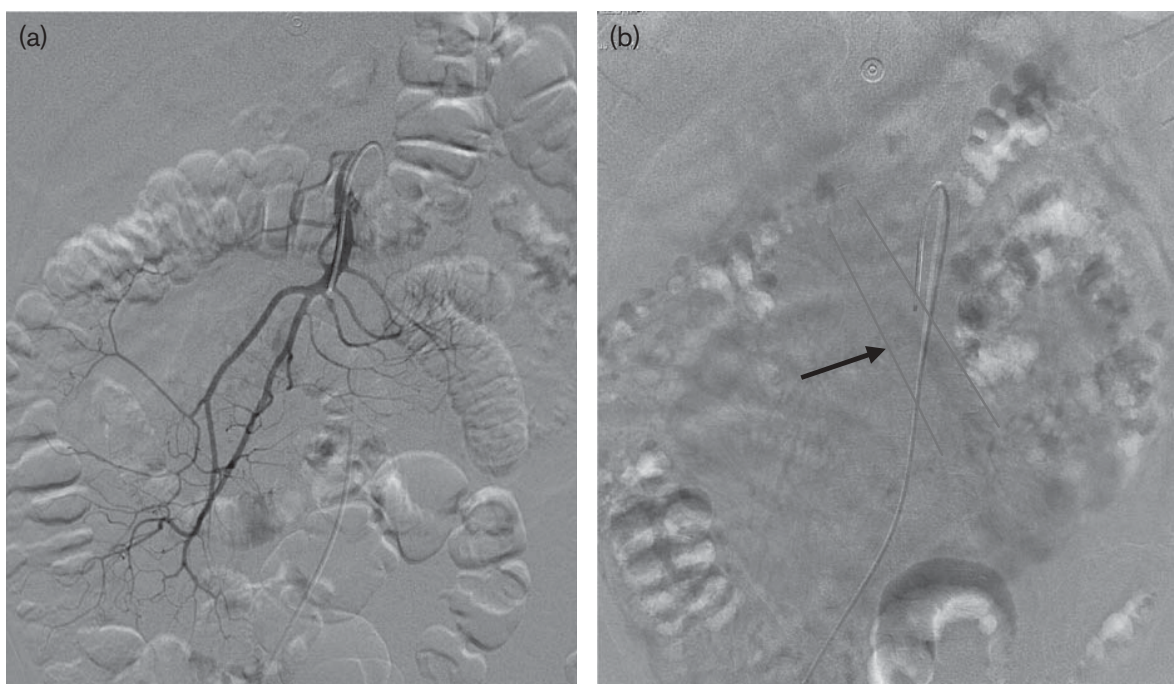

Angiography of the superior mesenteric artery at the moment of in-situ thrombolysis (a). Superior mesenteric vein (arrow) after in-situ thrombolysis (b).

Fig. 3

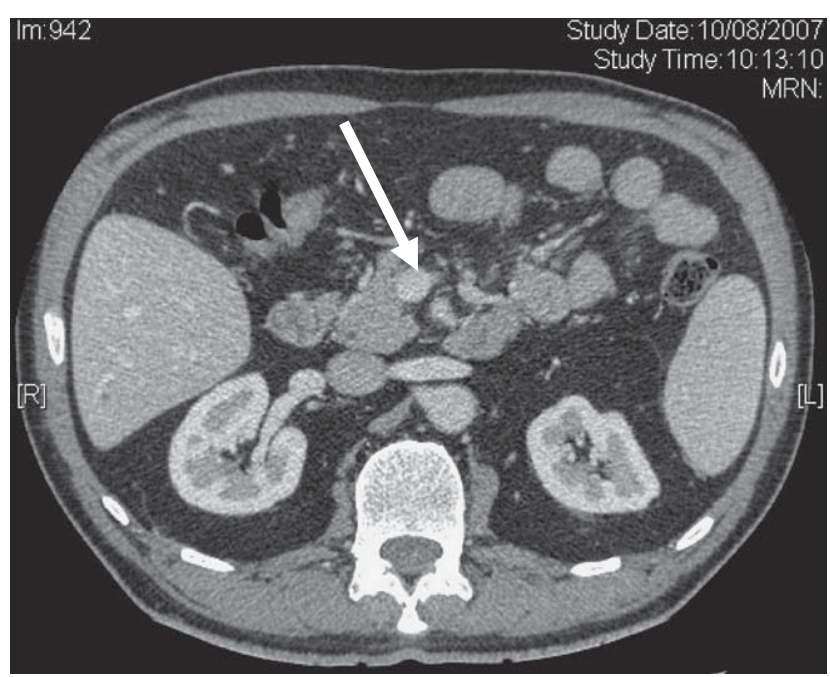

Complete recanalization (arrow) of the superior mesenteric vein.

We handle a multifactorial concept in which a certain trigger to thrombosis in a thrombophilic environment can initiate a thrombotic event $[23,24]$. Frequently a personal or family history of deep vein thrombosis or pulmonary embolism is present $[15,16]$. When peripheral blood results are normal, performing a bone marrow biopsy is required in the screening for occult myeloproliferative disorders. Recently, Janus kinase 2 mutation screening has become a front-line diagnostic test in the evaluation for occult myeloproliferative disorders when a PMVT is present $[23,25]$.
Table 2 Overview of etiology of portomesenteric vein thrombosis

Systemic factors: thrombophilia

Inherited (congenital)

Mutation of factor V Leiden

Mutation of prothrombin (factor II)

Protein $\mathrm{C}$ and $\mathrm{S}$ deficiency

Antithrombin III deficiency

Acquired: myeloproliferative syndromes (polycythemia vera, essential

thrombocythemia); pregnancy, oral contraception, neoplasm,

antiphospholipid syndrome, infections (CMV), ..

Local factors

Inflammation

Pancreatitis

Inflammatory bowel disease

Cholecystitis

Diverticulitis

Appendicitis

Peritonitis and intra-abdominal abscess

Intra-abdominal trauma

Abdominal surgery (e.g. splenectomy, colectomy, liver transplantation)

Neoplasm (e.g. pancreascarcinoma)

Cirrhosis with portal hypertension

Sclerotherapy for esophageal varices

Idiopathic ${ }^{\mathrm{a}}$

CMV, cytomegalovirus.

${ }^{a}$ Twenty percent (this percentage will further decrease with the discovery and increasing knowledge of genetic thrombophilia and better diagnostic tools and understanding such as the Janus kinase 2 mutation in screening for occult myeloproliferative disorders).

In this case report, we describe acute CMV infection as another factor triggering portomesenteric thrombosis [3]. Studies with rats had shown a change in the production of eicosanoids and increased procoagulant effects after infection with CMV [26,27]. Several pathophysiological mechanisms are assumed as an explanation for the thrombophilia, mostly based on 'in-vitro' studies. First, CMV has an endothelial cell tropism with damage of the 
vascular endothelium [28]. An inflammated endothelium shows a higher level of adhesion of circulating blood cells $[29,30]$. A rearrangement of phospholipids in the cell membrane after a CMV infection is responsible for a facilitation of interaction with coagulation factors [29-31]. Second, the adhesion of platelets and leukocytes (leukotropism) is changed by a modulation of the expression of adhesion molecules [intercellular adhesion molecule, E-selectin,... ] [7]. Third, the synthesis of lupus anticoagulans and factor VIII can be induced [8]. Antiphospholipid antibodies (anticardiolipin antibodies and lupus coagulans) can appear after several infections (e.g. viral infections such as CMV) and show an affinity for anionic phospholipids [4,32]. Finally, a deficiency of protein $\mathrm{C}$ (a natural anticoagulans) is possible after infection with CMV, possibly because of consumption as protein $\mathrm{C}$ has anti-inflammatory properties [8].

As mentioned, there is convincing 'in-vitro' evidence for the procoagulant effect of CMV. In a more clinical context, GMV is known to cause thrombotic events in patients with an immunodeficient state such as infection with HIV or immunosuppressive therapy $[11,12]$. Prospective studies to investigate the exact role of CMV infection as a risk factor for arterial and venous thrombosis and especially in immunocompetent persons are needed. It is not known why the entity of 'PMVT' is more frequent as the topographic and typical clinical presentation of a primary CMV infection complicated with thrombosis. Some reports on deep vein thrombosis and secondary pulmonary embolism are also observed [4,9-13]. In general, most immunocompetent patients with a (primary) CMV infection have minimal or no symptoms. Typically, initial therapy consists of antipyretics. Starting an antiviral therapy is not indicated and if so, it is not clear what the doses and duration of therapy should be.

In general, the clinical presentation of a PMVT depends on the extent of the thrombus, the size of the affected vessel, and the depth of bowel wall ischemia [15]. Two compensatory mechanisms have a protective role for the liver as a dual-vessel supplied organ. The first is the 'arterial rescue' in which arterial vasodilation occurs, the second is the 'venous rescue' involving the rapid development of a collateral venous network $[2,19,23]$. Extension to the splenic or PV is possible. Kumar and Kamath [14] describe two entities: 'large-vessel MVT' (thrombosis of the SMV associated with thrombosis of the splenic and/or PV) and 'small-vessel MV'T' (thrombosis of the smaller branches of the SMV). Less frequently, there is bowel infarction in large-vessel MVT, but when it occurs the extent of affected bowel is greater because of extension of thrombus to venous arcades and vasa recta.

Patients can have acute onset of symptoms. An atypical presentation with aspecific symptoms, such as abdominal pain, vomiting, and sometimes bloody diarrhea as a sign of intestinal ischemia, is also possible. An evolution to peritonitis makes urgent laparatomy mandatory, otherwise shock and death from multiple organ failure will occur $[33,34]$. Complaints can also develop in a subacute way (intra-abdominal discomfort for several days) or more chronically (complications of portal hypertension, e.g. variceal bleeding). It is important to know that up to $75 \%$ of patients have had symptoms for more than 2 days before medical care was searched [15,35]. Frequently, a diagnosis is missed and made at the moment of doing a laparotomy for acute abdomen or at autopsy $[16,36]$. Autopsy studies show the presence of superior MVT in $1.5 \%$ [16]. Several manners of medical imaging are observed [37]. Thrombotic material can be visualized with color Doppler ultrasonography. CT has a better sensitivity and also shows affected intestines and discloses other major intra-abdominal pathology such as pancreatitis. The role of conventional angiography as a gold standard for assessment of the splanchnic vasculature is replaced by CT and MRI but remains superior for demonstrating small mesenteric branch vessels [37].

The importance of a quick diagnosis and treatment must be stressed as the risk of intestinal ischemia and infarction increases with delay of treatment [33]. Second, the chance for recanalization is optimized. Until now, anticoagulation (heparin infusion, low molecular weight heparins, and oral anticoagulation) was the gold standard in the treatment of an acute PMVT. The absence of clinical, endoscopic or radiological evidence of portal hypertension is suggestive for the more recent onset of the PMVT [22]. The aim is a maximal repermeation and prevention of reoccurrence of thrombosis at short term. Early anticoagulation for acute PMVT can achieve complete or partial recanalization in 50 and $40 \%$ of patients, respectively $[1,16,18]$ Prevention of portal hypertension at long term is fundamental, as esophageal varices show a bleeding risk [22].

The use of a more aggressive approach can be defended in certain cases, for example, in the presence of an evolution to an acute abdomen as present in our case [38]. This approach includes systemic thrombolysis on the one hand and in-situ thrombolysis on the other. Local instillation of a thrombolytic agent achieves high local drug concentrations permitting efficient treatment [38]. On account of its fibrin specificity and more efficacious dissolution of thrombi, rt-PA is more frequently used [39-41]. In-situ administration is possible in a transhepatic (retrograde) or transarterial (antegrade) way $[40,42,43]$. In the latter approach the SMA is instillated with a local thrombolyticum [43]. The bleeding risk is less and the transarterial administration is preferred when treating a MVT $[38,44]$. The transarterial infusion enables the thrombolytic agent to permeate the capil- 
laries and small venules $[16,44]$. In literature, the dose of rt-PA is between 0.25 and $0.50 \mathrm{mg} / \mathrm{h} \mathrm{[38].} \mathrm{In} \mathrm{our} \mathrm{case} \mathrm{the}$ administered dose was higher, namely $1.8-3 \mathrm{mg} / \mathrm{h}$. It is usually recommended to use intravenous heparin for at least $24 \mathrm{~h}$ in maintaining vessel patency and preventing reocclusion [41].

One could think that there is a possible greater bleeding risk when anticoagulation or thrombolysis are initiated. In a study from Condat and Valla, anticoagulant therapy in 84 out of 136 patients did not increase the risk or severity of bleeding [19,45]. Repermeation prevents intestinal ischemia in the short term and an evolution to portal hypertension in the long term $[19,45]$. This outweighs the associated risks and indicates a potential favorable benefit-risk ratio for anticoagulation. In a retrospective study of 20 patients receiving thrombolysis via the SMA or the PV, the overall complication rate was $60 \%$ with bleeding or decreased hematocrit level for which transfusion was mandatory (in nine out of 20 patients); but except for one patient - who died - the other eight patients, for whom transfusion was necessary, improved. In that study there was a complete recanalization in $15 \%$, partial result in 60\%, and no effect in 25\% [38]. Nevertheless, there are several limitations in the correct interpretation as a control group (who did not receive treatment or oral anticoagulation alone), limitations in randomization, and uniformity of diagnosis and treatment are lacking. Patients for which thrombolysis is initiated more frequently have a severe pathology. For this reason a prospective study in which a uniform strategy is present, is mandatory.

Oral anticoagulation is not only useful in the treatment of an acute thrombosis, but also in preventing recurrent thrombosis [45]. The therapy is usually maintained for a period of 6-12 months when a specific trigger is apparent and when there is no evidence for a prothrombotic disorder (i.e. underlying hypercoagulable state) for which a lifelong therapy should be recommended [19]. The optimal duration of anticoagulant therapy for prevention of recurrent thrombosis in patients with antiphospholipid antibodies is unknown. The decision regarding the duration of anticoagulation can be influenced by the type of antiphospholipid antibody (anticardiolipin antibody, lupus anticoagulans, or both) and a persisting antibody response [46].

Although a surgical intervention is associated with a high mortality because of a high number of reinterventions and postoperative complications, a close clinical and radiological follow-up is mandatory [38]. Surgery with resection of nonviable intestinal tissue is an option in the acute setting when there is evidence for underlying small bowel infarction and if needed a second look laparatomy can be necessary in reevaluation of the evolution at short term as bowel infarction has a high mortality rate, up to $50 \%$ [15].

\section{Conclusion}

When confronted with a patient with a viral syndrome and progressive abdominal pain because of MVT, it is useful to look for a possible CMV infection as predisposing factor to thrombosis as shown by several recent publications. Inversely, the index of suspicion for MVT when a patient with recent CMV infection develops abdominal pain should be high.

Whatever the etiology of the acute persistent PMVT, we emphasize and propose a more aggressive therapeutic approach in an attempt to create a patent vessel as shown in Discussion. Indeed, thrombolysis is indicated in selected cases: for example, when extensive portomesenteric thrombosis, a severe symptomatology (e.g. acute abdomen with signs of peritoneal tenderness), progression under anticoagulant therapy, or a clinical deterioration are present.

This is in contrast with only starting anticoagulation in the more traditional approach in the treatment of a PMVT. We also refer to thrombolysis as a well established therapy in ischemic coronary and cerebral disease. Not much information is available about the best choice of thrombolyticum, the route of administration and the duration of the therapy. The same problem exists for therapy with oral anticoagulation. Clinical and prospective studies in determining guidelines for the treatment of an acute PMVT are needed.

\section{References}

1 Condat B, Pessione F, Denninger MH, Hillaire S, Valla D. Recent portal or mesenteric venous thrombosis: increased recognition and frequent recanalization on anticoagulant therapy. Hepatology 2000; 32:466-470.

2 Bayraktar Y, Harmanci O. Etiology and consequences of thrombosis in abdominal vessels. World J Gastroenterol 2006; 12:1165-1174.

3 Amitrano L, Guardascione MA, Scaglione M, Menchise A, Romano L, Balzano A. Acute portal and mesenteric thrombosis: unusual presentation of cytomegalovirus infection. Eur J Gastroenterol Hepatol 2006; 18:433-445.

4 Delbos V, Abgueguen P, Chennebault JM, Fanello S, Pichard E. Acute cytomegalovirus infection and venous thrombosis: role of antiphospholipid antibodies. J Infect 2007; 54:47-50.

5 Angelillo-Scherrer A. Portal vein thrombosis following a primary cytomegalovirus infection in a immunocompetent adult. Thromb Haemost 2006; 95:199-201.

6 Benoist S, Laisné MJ, Joly F, Boudiaf M, Panis Y, Valleur P. Cytomegalovirus infection as a cause of acute superior mesenteric vein thrombosis with jejunal infarction. Surgery 2003; 133:222-223.

7 Ofotokun I, Carlson C, Gitlin SD, Elta G, Singleton TP, Markovitz DM. Acute cytomegalovirus infection complicated by vascular thrombosis: a case report. Clin Infect Dis 2001; 32:983-986.

8 Lijfering WM, Sprenger HG, van Son WJ, van der Meer J. Mesenteric vein thrombosis associated with primary cytomegalovirus infection: a case report. Blood Coagul Fibrinolysis 2007; 18:509-511.

9 Bauduer F, Blanc A, Cordon B. Deep vein thrombosis and acute cytomegalovirus infection: case report and review of the literature. Blood Coagul Fibrinolysis 2003; 14:489-491.

10 Youd P, Main J, Jackson E. Cytomegalovirus infection and thrombosis: a causative association?. J Infect 2003; 46:141-143.

11 Abgueguen P, Delbos V, Chennebault JM, Payan C, Pichard E. Vascular thrombosis and acute cytomegalovirus infection in immunocompetent 
patients: report of 2 cases and literature review. Clin Infect Dis 2003; 36:134-139.

12 Rovery C, Granel B, Parola P, Foucault C, Brouqui P. Acute cytomegalovirus infection complicated by venous thrombosis: a case report. Ann Clin Microbiol Antimicrob 2005; 4:11.

13 Squizzato A, Ageno W, Cattaneo A, Brumana N. A case report and literature review of portal vein thrombosis associated with cytomegalovirus infection in immunocompetent patients. Clin Infect Dis 2007; 44:13-16.

14 Kumar S, Kamath PS. Acute superior mesenteric venous thrombosis: one disease or two? Am J Gastroenterol 2003; 98:1299-1304.

15 Kumar S, Sarr MG, Kamath PS. Mesenteric venous thrombosis. N Engl J Med 2001; 345:1683-1688.

16 Joh JH, Kim DI. Mesenteric and portal vein thrombosis: treated with early initiation of anticoagulation. Eur J Vasc Endovasc Surg 2005; 29:204-208.

17 Sreenarasimhaiah J. Diagnosis and management of intestinal ischaemic disorders. Br Med J 2003; 326:1372-1376.

18 Valla D, Condat B. Nonmalignant portal vein thrombosis in adults. Nat Clin Pract Gastroenterol Hepatol 2006; 3:505-515.

19 Valla D, Condat B. Portal vein thrombosis in adults: pathophysiology, pathogenesis and management. J Hepatol 2000; 32:865-871.

20 Condat B, Valla D. Management of portal vein thrombosis. Gastroenterol Clin Biol 1999; 23:1210-1214.

21 Wang JT, Zhao HY, Liu YL. Portal vein thrombosis. Hepatobiliary Pancreat Dis Int 2005; 4:515-518.

22 Webster GJM, Burroughs AK, Riordan SM. Review article: portal vein thrombosis - new insights into aetiology and management. Aliment Pharmacol Ther 2005; 21:1-9.

23 Harmanci O, Bayraktar Y. Portal hypertension due to portal venous thrombosis: etiology, clinical outcomes. World J Gastroenterol 2007; 13:2535-2540.

24 Rosendaal FR. Venous thrombosis: a multicausal disease. Lancet 1993; 353:1167-1173.

25 Colaizzo D, Amitrano L, Tiscia GL, Scenna G, Grandone E, Guardascione MA et al. The JAK2 V617F mutation frequently occurs in patients with portal and mesenteric venous thrombosis. J Thromb Haemost 2007; 5:55-61.

26 Engels W, Lemmens PJMR, Muller AD, Van Dam-Mieras MCE, Hornstra G. The effect of viral infection on eicosanoid formation and procoagulant activity of rat peritoneal macrophages. Eicosanoids 1992; 5:73-80.

27 Bouwman JJM, Visseren FLJ, Bosch MC, Bouter KP, Diepersloot RJA. Procoagulant and inflammatory response of virus-infected monocytes. Eur $J$ Clin Invest 2002; 32:759-766.

28 Van Dam-Mieras MCE, Bruggeman CA, Muller AD, Debie WHM, Zwaal RFA. Induction of endothelial cell procoagulant activity by cytomegalovirus infection. Thromb Res 1987; 47:69-75.

29 Van Geelen AGM, Slobbe-van Drumen MEP, Muller AD, Bruggemand CA, Van Dam-Mieras MCE. Membrane related effects in endothelial cells induced by human cytomegalovirus. Arch Virol 1995; 140:1601-1612.

30 Squizzato A, Gerdes VEA, Büller HR. Effects of human cytomegalovirus infection on the coagulation system. Thromb Haemost 2005; 93:403-410.

31 Van Dam-Mieras MCE, Muller AD, van Hinsbergh VWM, Mullers WJHA, Bomans $\mathrm{PHH}$, Bruggeman CA. The procoagulant response of cytomegalovirus infected endothelial cells. Thromb Haemost 1992; 68:364-370.

32 Labarca JA, Rabaggliati RM, Radrigan FJ. Antiphospholipid syndrome associated with cytomegalovirus infection: case report and review. Clin Infect Dis 1997; 24:187-200.

33 Tateishi A, Mitsui H, Oki T, Morishita J, Maekawa H, Yahagi N, et al. Extensive mesenteric vein and portal vein thrombosis successfully treated by thrombolysis and anticoagulation. J Gastroenterol Hepatol 2001; 16: 1429-1433.

34 Espeel B, Gérard C, Mansvelt B, Bertrand C, Vermonden J. Extensive mesenteric venous thrombosis treatment by regional thrombolysis. Ann Fr Anesth Reanim 2005; 24:274-277.

35 Rhee RY, Gloviczki P, Mendonca CT, Petterson TM, Serry RD, Sarr MG, et al. Mesenteric venous thrombosis: still a lethal disease in the 1990's. J Vasc Surg 1994; 20:688-697.

36 Morasch MD, Ebaugh JL, Chiou AC, Matsumura JS, Pearce WH, Yao JS. Mesenteric venous thrombosis: a changing clinical entity. J Vasc Surg 2001; 34:680-684.

37 Bradbury MS, Kavanagh PV, Chen MY, Weber TM, Bechtold RE. Noninvasive assessment of portomesenteric venous thrombosis: current concepts and imaging strategies. J Comput Assist Tomogr 2002; 26 : 392-404.

38 Hollingshead M, Burke CT, Mauro MA, Weeks SM, Dixon RG, Jaques PF. Transcatheter thrombolytic therapy for acute mesenteric and portal vein thrombosis. J Vasc Interv Radiol 2005; 16:651-661.

39 Kozuch PL, Brandt LJ. Review article: diagnosis and management of mesenteric ischaemia with an emphasis on pharmacotherapy. Aliment Pharmacol Ther 2005; 21:201-215.

40 Guglielmi A, Fior F, Halmos O, Veraldi GF, Rossaro L, Ruzzenente A, et al. Transhepatic fibrinolysis of mesenteric and portal vein thrombosis in a patient with ulcerative colitis: a case report. World J Gastroentero/ 2005; 11:2035-2038.

41 Hrsitc I, Kalauz M, Cukovic-Cavka S, Ostojic R, Banfic L, Vucelic B. Treatment of extensive subacute portal, mesenteric and ileocolic vein thrombosis with recombinant tissue plasminogen activator. Blood Coagul Fibrinolysis 2007; 18:581-583.

42 Bradbury MS, Kavanagh PV, Bechtold RE, Chen MY, Ott DJ, Regan JD, et al. Mesenteric venous thrombosis: diagnosis and non-invasive imaging. Radiographics 2002; 22:527-541.

43 Antoch G, Taleb N, Hansen O, Stock W. Transarterial thrombolysis of portal and mesenteric vein thrombosis: a promising alternative to common therapy. Eur J Vasc Endovasc Surg 2001; 21:471-472.

44 Henao EA, Bohannon WT, Silva MB. Treatment of portal venous thrombosis with selective superior mesenteric artery infusion of recombinant tissue plasminogen activator. J Vasc Surg 2003; 38:1411-1415.

45 Condat B, Pessione F, Hillaire S, Denninger MH, Guillin MC, Poliquin M, et al. Current outcomes of portal vein thrombosis in adults: risk and benefit of anticoagulant therapy. Gastroenterology 2001; 120:490-497.

46 Lim W, Crowther MA, Eikelboom JW. Management of antiphospholipid antibody syndrome: a systematic review. J Am Med Assoc 2006; 295:1050-1057. 